Pacific

Journal of

Mathematics

FUSION PRODUCTS AND TOROIDAL ALGEBRAS

Deniz Kus and Peter Littelmann 


\title{
FUSION PRODUCTS AND TOROIDAL ALGEBRAS
}

\author{
Deniz Kus AND Peter Littelmann
}

\begin{abstract}
We study the category of finite-dimensional bigraded representations of toroidal current algebras associated to finite-dimensional complex simple Lie algebras. Using the theory of graded representations for current algebras, we construct in different ways objects in that category and prove them to be isomorphic. As a consequence we obtain generators and relations for certain types of fusion products, including the $\boldsymbol{N}$-fold fusion product of $V(\lambda)$. This result shows that the fusion product of these types is independent of the chosen parameters, proving a special case of a conjecture by Feigin and Loktev. Moreover, we prove a conjecture by Chari, Fourier and Sagaki on truncated Weyl modules for certain classes of dominant integral weights and show that they are realizable as fusion products. In the last section we consider the case $\mathfrak{g}=\mathfrak{s l}_{2}$ and compute a PBW type basis for truncated Weyl modules of the associated current algebra.
\end{abstract}

\section{Introduction}

Let $\mathfrak{g}$ be a finite-dimensional complex simple Lie algebra with highest $\operatorname{root} \theta$. The current algebra $\mathfrak{g}[t]$ associated to $\mathfrak{g}$ is the algebra of polynomial maps $\mathbb{C} \rightarrow \mathfrak{g}$; equivalently, it is the complex vector space $\mathfrak{g} \otimes \mathbb{C}[t]$ with Lie bracket the $\mathbb{C}[t]$ bilinear extension of the Lie bracket on $\mathfrak{g}$. The toroidal current algebra $\mathfrak{g}[t, u]$ associated to $\mathfrak{g}$ is the algebra of polynomial maps $\mathbb{C}^{2} \rightarrow \mathfrak{g}$ and can be identified with the complex vector space $\mathfrak{g} \otimes \mathbb{C}[t, u]$ with similar Lie bracket. The Lie algebra $\mathfrak{g}[t]$ is graded by the nonnegative integers, where the $r$-th graded component is $\mathfrak{g} \otimes t^{r}$ and $\mathfrak{g}[t, u]$ is bigraded by pairs of nonnegative integers, where the $(r, s)$-th graded component is $\mathfrak{g} \otimes t^{r} u^{s}$. We are interested in the category of finite-dimensional graded representations of $\mathfrak{g}[t]$ and finite-dimensional bigraded representations of $\mathfrak{g}[t, u]$. The former category contains a large number of interesting objects, for example local Weyl modules (see for instance [Chari et al. 2014b; Chari and Pressley 2001; Fourier and Littelmann 2007; Fourier et al. 2012]), g-stable Demazure modules (see [Chari et al. 2014c; Fourier and Littelmann 2006; 2007]) and fusion products.

This work has been partially supported by the DFG Priority Program SPP 1388 (Representation Theory) and SFB/TR 12 (Symmetries and Universality in Mesoscopic Systems).

MSC2010: primary 17B67; secondary 17B10.

Keywords: fusion products, toroidal algebras, Demazure modules. 
The latter class of representations was introduced in a paper by Feigin and Loktev [1999]: given finite-dimensional cyclic $\mathfrak{g}[t]$-modules $\mathrm{V}_{1}, \ldots, \mathrm{V}_{N}$ with cyclic vectors $v_{1}, \ldots, v_{N}$ and a tuple of pairwise distinct complex numbers $z=$ $\left(z_{1}, \ldots, z_{N}\right)$ one can define a filtration on the tensor product $\mathrm{V}_{1}^{z_{1}} \otimes \cdots \otimes \mathrm{V}_{N}^{z_{N}}$ and build the associated graded space with respect to this filtration. This space is called the fusion product and is denoted by $\mathrm{V}_{1}^{z_{1}} * \cdots * \mathrm{~V}_{N}^{z_{N}}$, where $\mathrm{V}^{z}$ is a nongraded $\mathfrak{g}[t]$-module (see Section 3 for more details). The Feigin-Loktev conjecture states that under suitable conditions on $\mathrm{V}_{s}$ and $v_{s}$ the fusion product is independent of the chosen fusion parameters $z$. This conjecture has been proved for several classes of representations. For example it has been proved in [Chari and Loktev 2006; Chari and Pressley 2001; Fourier and Littelmann 2007; Naoi 2012] that the fusion product of local Weyl modules is again a local Weyl module and hence independent of the chosen parameters. Other examples are fusion products of Kirillov-Reshetikhin modules (see [Ardonne and Kedem 2007; Kedem 2011]) and fusion products of $\mathfrak{g}$-stable Demazure modules (see [Chari et al. 2014c; Fourier and Littelmann 2007; Kus and Venkatesh 2014]).

Another interesting class of $\mathfrak{g}[t]$-modules comprises those which are obtained as fusion products of finite-dimensional simple $\mathfrak{g}$-modules, where a $\mathfrak{g}$-module $\mathrm{V}$ is made into a $\mathfrak{g}[t]$-module by requiring $(\mathfrak{g} \otimes t \mathbb{C}[t]) \mathrm{V}=0$. Hence for any tuple $\left(\lambda_{1}, \ldots, \lambda_{N}\right)$ of dominant integral weights the fusion product $\mathrm{V}^{z_{1}}\left(\lambda_{1}\right) * \cdots * \mathrm{~V}^{z_{N}}\left(\lambda_{N}\right)$ can be defined and studied. For these types of representations the Feigin-Loktev conjecture has been proved in the case of $\mathfrak{s l}_{2}$ and in some other special cases (see for instance [Chari and Venkatesh 2015; Feigin and Feigin 2002; Feigin et al. 2004; Ravinder 2014]). Moreover, in the case of $\mathfrak{s l}_{2}$ a presentation for the fusion product $\mathrm{V}\left(k_{1}\right) * \cdots * \mathrm{~V}\left(k_{N}\right)$ has been established in terms of generators and relations of the enveloping algebra (see [Chari and Venkatesh 2015; Feigin and Feigin 2002]) and a PBW type basis has been computed [Chari and Venkatesh 2015]. An easy calculation shows that the aforementioned presentation can be greatly simplified if the highest weights are equal. In particular, $\mathrm{V}(k) * \cdots * \mathrm{~V}(k)$ is a cyclic $\boldsymbol{U}\left(\mathfrak{s l}_{2}[t]\right)$-module generated by a vector $v$ subject to the same relations as the highest weight vector of the local Weyl module $\mathrm{W}_{\text {loc }}(k N)$ with the only additional relation $\left(\mathfrak{s l}_{2} \otimes t^{N}\right) v=0$.

This paper is motivated by the idea of generalizing the above observation for arbitrary $\mathfrak{g}$ : Is the fusion product $\mathrm{V}^{z_{1}}(\lambda) * \cdots * \mathrm{~V}^{z_{N}}(\lambda)$ independent of the fusion parameters for arbitrary $\mathfrak{g}$ ? Is there a simple presentation considered as a $\boldsymbol{U}(\mathfrak{g}[t])$ module? Is the truncated Weyl module $\mathrm{W}(N \lambda, N)$ realizable as a fusion product? For the definition of truncated Weyl modules see Section 4A. In this paper we give a positive answer to these questions. Our approach is based on the theory of finite-dimensional bigraded modules for the toroidal current algebra $\mathfrak{g}[t, u]$. In particular we construct an associated graded version of a $\mathfrak{g}$-stable Demazure module $\operatorname{gr}_{t^{N}} \mathrm{~T}(\ell, N)$ and a bigraded version of a fusion product $\overline{\mathrm{D}^{u}(\ell, \ell \lambda)} * \cdots * \overline{\mathrm{D}^{u}(\ell, \ell \lambda)} *$ 
$\overline{\mathrm{D}^{u}\left(\ell, \ell \lambda+\lambda^{0}\right)}$ (for the precise definitions see Sections 3C and 3D) such that the zeroth graded space (with respect to the $u$-grading) of the second construction is isomorphic to the fusion product of finite-dimensional simple $\mathfrak{g}$-modules. Our first result is the following; for the precise definition of the ingredients see Section 3. We remark that if $\lambda^{0} \neq 0$, then the Lie algebra $\mathfrak{g}$ is assumed to be classical or $\mathrm{G}_{2}$; for $\lambda^{0}=0$ there is no restriction on $\mathfrak{g}$.

Theorem. Let $\ell \in \mathbb{N}, \lambda$ be a dominant integral coweight and $\lambda^{0}$ be a dominant integral weight such that $\lambda^{0}\left(\theta^{\vee}\right) \leq \ell$. We have an isomorphism of $\boldsymbol{U}(\mathfrak{g}[t, u])$ modules

$$
\operatorname{gr}_{t^{N}} \mathrm{~T}(\ell, N) \cong \overline{\mathrm{D}^{u}(\ell, \ell \lambda)} * \cdots * \overline{\mathrm{D}^{u}(\ell, \ell \lambda)} * \overline{\mathrm{D}^{u}\left(\ell, \ell \lambda+\lambda^{0}\right)} .
$$

Our second result gives a connection to truncated Weyl modules, where the first part is a direct consequence of the previous theorem and the second part proves a special case of a conjecture by Chari, Fourier and Sagaki. Again for the precise definition of the ingredients see Section 4A.

Theorem. Let $\ell \in \mathbb{N}, \lambda$ be a dominant integral coweight and $\lambda^{0}$ be a dominant integral weight such that $\lambda^{0}\left(\theta^{\vee}\right) \leq \ell$.

(1) The fusion product $\mathrm{V}(\ell \lambda)^{*(N-1)} * \mathrm{~V}\left(\ell \lambda+\lambda^{0}\right)$ is independent of the fusion parameters.

(2) If $\lambda^{0}\left(\theta^{\vee}\right) \leq 1$ and $\left|N \lambda+\lambda^{0}\right| \geq N$, then

$$
\mathrm{W}\left(N \lambda+\lambda^{0}, N\right) \cong \mathrm{V}(\lambda)^{*(N-1)} * \mathrm{~V}\left(\lambda+\lambda^{0}\right) .
$$

As a special case of the previous theorem we can choose $\ell=1$ and $\lambda^{0}=0$. This yields that the $N$-fold fusion product of $V(\lambda)$ is independent of the fusion parameters for any dominant integral coweight $\lambda$. The second part of the theorem shows that the $N$-fold fusion product of $V(\lambda)$ has a remarkably simple presentation.

In Sections 4C and 4D we deal with the case of $\mathfrak{s l}_{2}$ and prove that the truncated Weyl module is realizable as a fusion product. Moreover, we compute a PBW type basis for truncated Weyl modules which differs from the basis described in [Chari and Venkatesh 2015, Section 6]. For the precise definition of the set $S\left(k^{N-j},(k+1)^{j}\right)$ see Section 4B.

Theorem. Let $m \in \mathbb{Z}_{+}$and write $m=k N+j, 0 \leq j<N$.

(1) We have an isomorphism of $\boldsymbol{U}\left(\mathfrak{s l}_{2} \otimes \mathbb{C}[t] / t^{N}\right)$-modules

$$
\mathrm{W}(m, N) \cong V(k)^{*(N-j)} * V(k+1)^{* j} .
$$

(2) A PBW type basis of $\mathrm{W}(m, N)$ is given by

$$
\left\{\left(x_{-\alpha} \otimes 1\right)^{i_{0}} \cdots\left(x_{-\alpha} \otimes t^{N-1}\right)^{i_{N-1}} w_{m, N} \mid\left(i_{0}, \ldots, i_{N-1}\right) \in S\left(k^{N-j},(k+1)^{j}\right)\right\} .
$$


Our paper is organized as follows. Section 2 establishes the basic notation needed in the rest of the paper. In Section 3, we construct in different ways two bigraded modules and prove them to be isomorphic. As a consequence we obtain that the fusion product is independent of the chosen parameters. In Section 4, we give some applications regarding the conjecture on truncated Weyl modules and compute a PBW type basis.

\section{Preliminaries}

2A. Throughout the paper $\mathbb{C}$ denotes the field of complex numbers, $\mathbb{Z}$ the ring of integers, $\mathbb{Z}_{+}$the set of nonnegative integers and $\mathbb{N}$ the set of positive integers. Given any complex Lie algebra $\mathfrak{a}$ we let $\boldsymbol{U}(\mathfrak{a})$ be the universal enveloping algebra of $\mathfrak{a}$. Further, let $\mathfrak{a}[t]$ be the Lie algebra of polynomial maps from $\mathbb{C}$ to $\mathfrak{a}$ with the obvious pointwise Lie bracket:

$$
[x \otimes f, y \otimes g]=[x, y] \otimes f g, \quad x, y \in \mathfrak{a}, f, g \in \mathbb{C}[t] .
$$

The Lie algebra $\mathfrak{a}[t]$ and its universal enveloping algebra inherit a grading from the degree grading of $\mathbb{C}[t]$; thus an element $a_{1} \otimes t^{r_{1}} \cdots a_{s} \otimes t^{r_{s}}, a_{j} \in \mathfrak{a}, r_{j} \in \mathbb{Z}_{+}$for $1 \leq j \leq s$, will have grade $r_{1}+\cdots+r_{s}$. We shall be interested in $\mathbb{Z}$-graded modules $V=\bigoplus_{s \in \mathbb{Z}} V[s]$ for $\mathfrak{a}[t]$.

2B. We refer to [Kac 1990] for the general theory of affine Lie algebras. Let $\mathfrak{g}$ be a finite-dimensional complex simple Lie algebra and $\hat{\mathfrak{g}}$ be the corresponding untwisted affine algebra. We fix $\mathfrak{h} \subset \hat{\mathfrak{h}}$ Cartan subalgebras of $\mathfrak{g}$ and $\hat{\mathfrak{g}}$, respectively, and denote by $R$ the set of roots of $\mathfrak{g}$ with respect to $\mathfrak{h}$ and by $\widehat{R}$ the set of roots of $\hat{\mathfrak{g}}$ with respect to $\hat{\mathfrak{h}}$. The corresponding sets of positive and negative roots are denoted as usual by $R^{ \pm}$and $\widehat{R}^{ \pm}$, respectively. We fix a basis $\Delta=\left\{\alpha_{1}, \ldots, \alpha_{n}\right\}$ for $R$ such that $\widehat{\Delta}=\Delta \cup\left\{\alpha_{0}\right\}$ is a basis for $\widehat{R}$. For $\alpha \in \widehat{R}$, let $\alpha^{\vee}$ be the corresponding coroot. We fix $d \in \hat{\mathfrak{h}}$ such that $\alpha_{0}(d)=1$ and $\alpha_{i}(d)=0$ for $i \neq 0$; $d$ is called the scaling element and it is unique modulo the center of $\hat{\mathfrak{g}}$. For $1 \leq i \leq n$, define $\omega_{i} \in \mathfrak{h}^{*}$ by $\omega_{i}\left(\alpha_{j}^{\vee}\right)=\delta_{i, j}$ for $1 \leq j \leq n$, where $\delta_{i, j}$ is Kronecker's delta symbol. The element $\omega_{i}$ is the fundamental weight of $\mathfrak{g}$ corresponding to $\alpha_{i}^{\vee}$. Let $(\cdot, \cdot)$ be the nondegenerate symmetric bilinear form on $\mathfrak{h}^{*}$ normalized so that the square length of a long root is two. For $\alpha \in R^{+}$we set

$$
d_{\alpha}=\frac{2}{(\alpha, \alpha)}, \quad d_{i}:=d_{\alpha_{i}} \text { for } 1 \leq i \leq n .
$$

The weight lattices $P$ and $P^{+}$are the $\mathbb{Z}$-span and $\mathbb{Z}_{+}$-span, respectively, of the fundamental weights. The coweight lattice $L$ is the sublattice of $P$ spanned by the 
elements $d_{i} \omega_{i}, 1 \leq i \leq n$, and the subset $L^{+}$is defined in the obvious way. For $\lambda \in P^{+}$we define

$$
|\lambda|=\sum_{i=1}^{n} \lambda\left(\alpha_{i}^{\vee}\right) \in \mathbb{Z}_{+} .
$$

2C. Given $\alpha \in \widehat{R}^{+}$let $\hat{\mathfrak{g}}_{\alpha} \subset \hat{\mathfrak{g}}$ be the corresponding root space; note that $\hat{\mathfrak{g}}_{\alpha} \subset \mathfrak{g}$ if $\alpha \in R$. For a real root $\alpha$ we denote by $x_{\alpha}$ a generator of $\hat{\mathfrak{g}}_{\alpha}$. The element $d$ defines a $\mathbb{Z}_{+}$-graded Lie algebra structure on $\mathfrak{g}[t]$ : for $\alpha \in \widehat{R}$ we say that $\mathfrak{g}_{\alpha}$ has grade $k$ if

$$
\left[d, x_{\alpha}\right]=k x_{\alpha}
$$

or, equivalently, if $\alpha(d)=k$. With respect to this grading, the zero homogeneous component of the current algebra is $\mathfrak{g}[t][0] \cong \mathfrak{g}$ and the subspace spanned by the positive homogeneous components is an ideal denoted by $\mathfrak{g}[t]_{+}$. We have a short exact sequence of Lie algebras

$$
0 \rightarrow \mathfrak{g}[t]_{+} \rightarrow \mathfrak{g}[t] \stackrel{\mathrm{ev}_{0}}{\longrightarrow} \mathfrak{g} \rightarrow 0 .
$$

Clearly the pull-back of any $\mathfrak{g}$-module $\mathrm{V}$ by ev $_{0}$ defines the structure of a graded $\mathfrak{g}[t]$-module on $\mathrm{V}$, and we denote this module by $\mathrm{ev}_{0}^{*} \mathrm{~V}$.

2D. For $\lambda \in P^{+}$, denote by $\mathrm{V}(\lambda)$ the simple finite-dimensional $\mathfrak{g}$-module generated by an element $v_{\lambda}$ with defining relations

$$
\mathfrak{n}^{+} v_{\lambda}=0, \quad \alpha_{i}^{\vee} v_{\lambda}=\lambda\left(\alpha_{i}^{\vee}\right) v_{\lambda}, \quad\left(x_{-\alpha_{i}}\right)^{\lambda\left(\alpha_{i}^{\vee}\right)+1} v_{\lambda}=0, \quad 1 \leq i \leq n .
$$

It is well known that $\mathrm{V}(\lambda) \cong \mathrm{V}(\mu)$ if and only if $\lambda=\mu$ and that any finite-dimensional $\mathfrak{g}$-module is isomorphic to a direct sum of modules $\mathrm{V}(\lambda), \lambda \in P^{+}$. If $\mathrm{V}$ is an $\mathfrak{h}$ semisimple $\mathfrak{g}$-module (in particular if $\operatorname{dim} \mathrm{V}<\infty$ ), we have

$$
\mathrm{V}=\bigoplus_{\mu \in \mathfrak{h}^{*}} \mathrm{~V}_{\mu}, \quad \mathrm{V}_{\mu}=\{v \in \mathrm{V} \mid h v=\mu(h) v, h \in \mathfrak{h}\},
$$

and we set wt $\mathrm{V}=\left\{\mu \in \mathfrak{h}^{*} \mid V_{\mu} \neq 0\right\}$. By our previous comments, for any $\lambda \in P^{+}$ we obtain a graded $\mathfrak{g}[t]$-module $\mathrm{ev}_{0}^{*} \mathrm{~V}(\lambda)$.

We also define the local Weyl module $\mathrm{W}_{\text {loc }}(\lambda)$, which is a finite-dimensional $\mathfrak{g}[t]$-module generated by an element $w_{\lambda}$ with defining relations

$$
\begin{array}{r}
\mathfrak{n}^{+}[t] w_{\lambda}=0, \quad\left(\alpha_{i}^{\vee} \otimes t^{s}\right) w_{\lambda}=\delta_{s, 0} \lambda\left(\alpha_{i}^{\vee}\right) w_{\lambda}, \quad\left(x_{-\alpha_{i}} \otimes 1\right)^{\lambda\left(\alpha_{i}^{\vee}\right)+1} w_{\lambda}=0 \\
\forall s \geq 0,1 \leq i \leq n .
\end{array}
$$

For more details regarding the theory of local Weyl modules see [Chari et al. 2014b; Chari and Loktev 2006; Chari and Pressley 2001; Fourier et al. 2012; Fourier and Kus 2013; Fourier and Littelmann 2007; Naoi 2012]. 
2E. We recall a general construction from [Feigin and Loktev 1999]. Let $\boldsymbol{U}(\mathfrak{g}[t])[k]$ be the homogeneous component of degree $k$ (with respect to the grading induced by $d$ ) and recall that it is a $\mathfrak{g}$-module for all $k \in \mathbb{Z}_{+}$. Suppose now that we are given a $\mathfrak{g}[t]$-module $\mathrm{V}$ which is generated by $v$. Define an increasing filtration $0 \subset \mathrm{V}^{0} \subset \mathrm{V}^{1} \subset \cdots$ of $\mathfrak{g}$-submodules of $\mathrm{V}$ by

$$
\mathrm{V}^{k}=\bigoplus_{s=0}^{k} \boldsymbol{U}(\mathfrak{g}[t])[s] v .
$$

The associated graded vector space gr $\mathrm{V}$ admits an action of $\mathfrak{g}[t]$ given by

$$
x\left(v+\mathrm{V}^{k}\right)=x v+\mathrm{V}^{k+s}, \quad x \in \mathfrak{g}[t][s], v \in \mathrm{V}^{k+1} .
$$

Furthermore, gr V is a cyclic $\mathfrak{g}[t]$-module with cyclic generator $\bar{v}$, the image of $v$ in gr $\mathrm{V}$. Given a $\mathfrak{g}[t]$-module $\mathrm{V}$ and $z \in \mathbb{C}$, let $\mathrm{V}^{z}$ be the $\mathfrak{g}[t]$-module with action

$$
\left(x \otimes t^{r}\right) w=\left(x \otimes(t+z)^{r}\right) w, \quad x \in \mathfrak{g}, w \in \mathrm{V}, r \in \mathbb{Z}_{+} .
$$

Starting with finite-dimensional cyclic $\mathfrak{g}[t]$-modules $\mathrm{V}_{1}, \ldots, \mathrm{V}_{N}$ with cyclic vectors $v_{1}, \ldots, v_{N}$ and a tuple of pairwise distinct complex numbers $z=\left(z_{1}, \ldots, z_{N}\right)$, the fusion product is defined to be $\mathrm{V}^{z_{1}} * \cdots * \mathrm{~V}^{z_{N}}:=\operatorname{gr}\left(\mathrm{V}^{z_{1}} \otimes \cdots \otimes \mathrm{V}^{z_{N}}\right)$. It was proved in [Feigin and Loktev 1999] that the tensor product $\mathrm{V}^{z_{1}} \otimes \cdots \otimes \mathrm{V}^{z_{N}}$ is cyclic and generated by $v_{1} \otimes \cdots \otimes v_{N}$. Clearly the definition of the fusion product depends on the parameters $z_{s}, 1 \leq s \leq N$. However, it was conjectured in that paper (and proved in special cases; see [Chari and Loktev 2006; Feigin and Feigin 2002; Feigin and Loktev 1999; Fourier and Littelmann 2007; Kus and Venkatesh 2014], for example) that under suitable conditions on $\mathrm{V}_{s}$ and $v_{s}$, the fusion product is independent of the choice of the complex numbers. In this paper we cover another class of representations, where the construction of the fusion product is independent of the parameters. To keep the notation as simple as possible we almost always omit the parameters in the notation for the fusion product and write $\mathrm{V}_{1} * \cdots * \mathrm{~V}_{N}$ for $\mathrm{V}_{1}^{z_{1}} * \cdots * \mathrm{~V}_{N}^{z_{N}}$.

\section{Filtrations and bigraded modules}

The aim of this section is to construct two finite-dimensional bigraded modules in different ways and prove them to be isomorphic. The advantage of this construction is that a comparison of the zeroth graded components leads to a realization of the fusion product associated to rectangular partitions.

3A. Let us start with our first construction. Let $\lambda \in P^{+}$and $\ell \in \mathbb{N}$. The $\mathfrak{g}$-stable Demazure module $\mathrm{D}(\ell, \lambda)$ is a finite-dimensional submodule of a level $\ell$ highest weight representation for the affine algebra $\hat{\mathfrak{g}}$. For these representations, generators 
and relations are known if we consider them as $\boldsymbol{U}(\mathfrak{g}[t])$-modules; see [Fourier and Kus 2013; Fourier and Littelmann 2007; Mathieu 1988] for more details. We remark that these relations are greatly simplified for Demazure modules for untwisted affine algebras in [Chari and Venkatesh 2015] and for twisted affine algebras in [Kus and Venkatesh 2014]. For instance, one can use these simplified relations to see directly that level one Demazure modules are isomorphic to local Weyl modules for simply laced affine algebras and twisted affine algebras, initially proved in [Fourier and Littelmann 2007] and [Chari et al. 2014b; Fourier and Kus 2013], respectively. We recall the simplified presentation of $\mathfrak{g}$-stable Demazure modules. Write

$$
\lambda\left(\beta^{\vee}\right)=\left(p_{\beta}-1\right) d_{\beta} \ell+m_{\beta}, \quad 0<m_{\beta} \leq d_{\beta} \ell, \text { for } \beta \in R^{+} .
$$

Proposition 3.1. The Demazure module $\mathrm{D}(\ell, \lambda)$ is isomorphic to the cyclic $\boldsymbol{U}(\mathfrak{g}[t])$ module generated by a vector $v \neq 0$ subject to the following relations:

$$
\begin{aligned}
& \mathfrak{n}^{+}[t] v=0, \quad\left(h \otimes t^{s}\right) v=\delta_{s, 0} \lambda(h) v \quad \forall h \in \mathfrak{h}, s \geq 0, \\
& \left(x_{-\beta} \otimes 1\right)^{\lambda\left(\beta^{\vee}\right)+1} v=0, \quad\left(x_{-\beta} \otimes t^{p_{\beta}}\right) v=0 \quad \forall \beta \in R^{+}, \\
& \left(x_{-\beta} \otimes t^{p_{\beta}-1}\right)^{m_{\beta}+1} v=0 \quad \forall \beta \in R^{+} \text {such that } m_{\beta}<d_{\beta} \ell .
\end{aligned}
$$

We can decompose $\mathrm{D}(\ell, \lambda)$ into simple finite-dimensional $\mathfrak{g}$-modules. We remark that the vector $v$ in Proposition 3.1 corresponds to the highest weight vector of $\operatorname{ev}_{0}^{*} \mathrm{~V}(\lambda)$ in the $\mathfrak{g}$-module decomposition of $\mathrm{D}(\ell, \lambda)$. We call it a highest weight vector of the module.

3B. We are concerned with Demazure modules of the form $\mathrm{D}\left(\ell, \ell N \lambda^{1}+\lambda^{0}\right)$, where $\lambda^{1} \in L^{+}$and $\lambda^{0} \in P^{+}$such that $\lambda^{0}\left(\theta^{\vee}\right) \leq \ell$. For the rest of this paper we assume that either $\lambda^{0}=0$ and $\mathfrak{g}$ is arbitrary or $\lambda^{0} \neq 0$ and $\mathfrak{g}$ is of classical type or $\mathrm{G}_{2}$. By the results of [Chari et al. 2014c; Fourier and Littelmann 2007], the Demazure module $\mathrm{D}\left(\ell, \ell N \lambda^{1}+\lambda^{0}\right)$ is isomorphic to the fusion product of $N-1$ copies of the Demazure module $\mathrm{D}\left(\ell, \ell \lambda^{1}\right)$ with $\mathrm{D}\left(\ell, \ell \lambda^{1}+\lambda^{0}\right)$ :

$$
\mathrm{D}\left(\ell, \ell N \lambda^{1}+\lambda^{0}\right) \cong \mathrm{D}\left(\ell, \ell \lambda^{1}\right) * \cdots * \mathrm{D}\left(\ell, \ell \lambda^{1}\right) * \mathrm{D}\left(\ell, \ell \lambda^{1}+\lambda^{0}\right) .
$$

This decomposition holds for all fusion parameters $z=\left(z_{1}, \ldots, z_{N}\right)$ with $z_{i} \neq z_{j}$ for all $i \neq j$. We emphasize that the restriction on $\mathfrak{g}$ is only made because (3-5) is not proved for the remaining exceptional Lie algebras if $\lambda^{0}$ is nonzero. In other words, our results are applicable whenever we have such a fusion product decomposition. We will need the following lemma.

Lemma 3.2. Let $\beta$ be a positive root and $\lambda \in P^{+}$. We write $\theta-\beta=\sum_{j} \gamma_{j}$ as a sum of positive roots. Then we have

$$
\lambda\left(\beta^{\vee}\right)(\beta, \beta) \leq \lambda\left(\theta^{\vee}\right)(\theta, \theta) \quad \text { with equality if and only if } \lambda\left(\gamma_{j}^{\vee}\right)=0 \forall j .
$$


Proof. Since $\lambda$ is a dominant integral weight we have $\lambda\left(\beta^{\vee}\right) \geq 0$ for a positive root $\beta$. We obtain

$$
\theta^{\vee}=\left(\beta+\sum_{j} \gamma_{j}\right)^{\vee}=\frac{(\beta, \beta)}{2} \beta^{\vee}+\sum_{j} \frac{\left(\gamma_{j}, \gamma_{j}\right)}{2} \gamma_{j}^{\vee},
$$

which gives

$$
\lambda\left(\theta^{\vee}\right)(\theta, \theta)=(\beta, \beta) \lambda\left(\beta^{\vee}\right)+\sum_{j}\left(\gamma_{j}, \gamma_{j}\right) \lambda\left(\gamma_{j}^{\vee}\right) \geq(\beta, \beta) \lambda\left(\beta^{\vee}\right) .
$$

Note that equality is only possible if $\lambda\left(\gamma_{j}^{\vee}\right)=0$ for all $j$, since $\left(\gamma_{j}, \gamma_{j}\right)>0$.

By Lemma 3.2 and Equation (3-3) of Proposition 3.1 we get the following result. Corollary 3.3. $\left(x_{-\beta} \otimes t^{\left(\lambda^{1}\left(\theta^{\vee}\right)+1\right) N}\right) v=0$ for all roots $\beta \in R^{+}$.

Proof. Write $\left(\ell N \lambda^{1}+\lambda^{0}\right)\left(\beta^{\vee}\right)$ as in (3-1). Since $\lambda^{1} \in L^{+}$we have $m_{\beta}=d_{\beta} \ell$ if $\lambda^{0}\left(\beta^{\vee}\right)=0$ and $m_{\beta}=\lambda^{0}\left(\beta^{\vee}\right)$ else. Then $\left(x_{-\beta} \otimes t^{p_{\beta}}\right) v=0$ and

$$
p_{\beta}=N \frac{\lambda^{1}\left(\beta^{\vee}\right)}{d_{\beta}}+\frac{\lambda^{0}\left(\beta^{\vee}\right)-m_{\beta}}{d_{\beta} \ell}+1 \leq N\left(\lambda^{1}\left(\theta^{\vee}\right)+1\right) .
$$

Hence $\mathrm{D}\left(\ell, \ell N \lambda^{1}+\lambda^{0}\right)$ is a $\boldsymbol{U}\left(\mathfrak{g} \otimes \mathbb{C}[t] / t^{\left(\lambda^{1}\left(\theta^{\vee}\right)+1\right) N}\right)$-module.

3C. We define a decreasing filtration on $\boldsymbol{U}\left(\mathfrak{g} \otimes \mathbb{C}[t] / t^{\left(\lambda^{1}\left(\theta^{\vee}\right)+1\right) N}\right)$

$$
\mathrm{T}_{0}(N) \supseteq \mathrm{T}_{1}(N) \supseteq \mathrm{T}_{2}(N) \supseteq \cdots,
$$

with

$$
\begin{aligned}
& \mathrm{T}_{0}(N)=\boldsymbol{U}\left(\mathfrak{g} \otimes \mathbb{C}[t] / t^{\left(\lambda^{1}\left(\theta^{\vee}\right)+1\right) N}\right), \\
& \mathrm{T}_{j}(N)=\left(\mathfrak{g} \otimes t^{N} \mathbb{C}[t]\right) \mathrm{T}_{j-1}(N) \quad \text { for } j \geq 1,
\end{aligned}
$$

and study the induced decreasing filtration on our Demazure module given by $\mathrm{D}\left(\ell, \ell N \lambda^{1}+\lambda^{0}\right)=\mathrm{T}_{0}(N) v=: \mathrm{T}_{0}(\ell, N) \supseteq \mathrm{T}_{1}(N) v:=\mathrm{T}_{1}(\ell, N) \supseteq \mathrm{T}_{2}(\ell, N) \supseteq \cdots$.

To be consistent with the notation in [Feigin 2008], we refer to it as the $t^{N}$-filtration. Let $\mathrm{gr}_{t^{N}} \mathrm{~T}(N)$ and $\mathrm{gr}_{t^{N}} \mathrm{~T}(\ell, N)$, respectively, be the associated graded spaces

$$
\mathrm{gr}_{t^{N}} T(N)=\mathrm{T}_{0}(N) / \mathrm{T}_{1}(N) \oplus \mathrm{T}_{1}(N) / \mathrm{T}_{2}(N) \oplus \cdots
$$

and

$$
\operatorname{gr}_{t^{N}} \mathrm{~T}(\ell, N)=\mathrm{T}_{0}(\ell, N) / \mathrm{T}_{1}(\ell, N) \oplus \mathrm{T}_{1}(\ell, N) / \mathrm{T}_{2}(\ell, N) \oplus \cdots .
$$

Since $\mathrm{D}\left(\ell, \ell N \lambda^{1}+\lambda^{0}\right)$ is a module for $\boldsymbol{U}\left(\mathfrak{g} \otimes \mathbb{C}[t] / t^{\left(\lambda^{1}\left(\theta^{\vee}\right)+1\right) N}\right)$ we obtain that $\mathrm{gr}_{t^{N}} \mathrm{~T}(\ell, N)$ is a module for $\operatorname{gr}_{t^{N}} \mathrm{~T}(N)$. By the following lemma $\operatorname{gr}_{t^{N}} \mathrm{~T}(\ell, N)$ is also a module for the toroidal current algebra $\boldsymbol{U}\left(\mathfrak{g} \otimes \mathbb{C}[t, u] /\left\langle t^{N}, u^{\lambda^{1}\left(\theta^{\vee}\right)+1}\right\rangle\right)$. 
Lemma 3.4. We have an isomorphism of algebras

$$
\Psi: \mathrm{gr}_{t^{N}} \mathrm{~T}(N) \stackrel{\sim}{\longrightarrow} \boldsymbol{U}\left(\mathfrak{g} \otimes \mathbb{C}[t, u] /\left\langle t^{N}, u^{\lambda^{1}\left(\theta^{\vee}\right)+1}\right\rangle\right),
$$

where $\Psi\left(x \otimes t^{j N+s}\right)=x \otimes u^{j} t^{s}$ for $x \in \mathfrak{g}$ and $0 \leq s<N$.

Proof. The map $\Psi$ is clearly an isomorphism of vector spaces. In order to show that this map is an algebra homomorphism, we have to check that the naive way of defining $\Psi$ on a product of elements is well defined. Hence we will verify that

$$
\left(x \otimes u^{j} t^{s}\right)\left(y \otimes u^{i} t^{q}\right)-\left(y \otimes u^{i} t^{q}\right)\left(x \otimes u^{j} t^{s}\right)=[x, y] \otimes u^{i+j} t^{s+q}
$$

holds on the right-hand side whenever we have

$$
\left(x \otimes t^{j N+s}\right)\left(y \otimes t^{i N+q}\right)-\left(y \otimes t^{i N+q}\right)\left(x \otimes t^{j N+s}\right)=[x, y] \otimes t^{(i+j) N+(s+q)}
$$

on the left-hand side. This is obvious for $s+q<N$. Otherwise the variables $x \otimes u^{j} t^{s}$ and $y \otimes u^{i} t^{q}$ commute in $\boldsymbol{U}\left(\mathfrak{g} \otimes \mathbb{C}[t, u] /\left\langle t^{N}, u^{\lambda^{1}\left(\theta^{\vee}\right)+1}\right\rangle\right)$. By the definition of the associated graded space we also obtain that the variables $x \otimes t^{j N+s}$ and $y \otimes t^{i N+q}$ commute in $\operatorname{gr}_{t^{N}} \mathrm{~T}(N)$ since on the one hand

$$
\left(x \otimes t^{j N+s}\right)\left(y \otimes t^{i N+q}\right)-\left(y \otimes t^{i N+q}\right)\left(x \otimes t^{j N+s}\right) \in \mathrm{T}_{i+j}(N)
$$

and on the other hand

$$
[x, y] \otimes t^{(i+j) N+(s+q)} \in \mathrm{T}_{i+j+1}(N) .
$$

3D. Now we present a quite different construction of the module $\operatorname{gr}_{t^{N}} \mathrm{~T}(\ell, N)$. In fact, it is one of the main results of this paper that the two constructions give isomorphic modules. We start with the $(N-1)$-fold tensor product of Demazure modules $\mathrm{D}\left(\ell, \ell \lambda^{1}\right)$ with $\mathrm{D}\left(\ell, \ell \lambda^{1}+\lambda^{0}\right)$. The gambit: we switch the variables and consider now the current algebra $\mathfrak{g}[u]$ which operates on the Demazure modules $\mathrm{D}^{u}\left(\ell, \ell \lambda^{1}\right)$. We add the index $u$ to emphasize that here the algebra $\mathfrak{g}[u]$ is acting. We extend the action trivially to $\mathfrak{g}[t, u]$ and denote the corresponding module by $\overline{\mathrm{D}^{u}\left(\ell, \ell \lambda^{1}\right)}$; i.e., $\mathfrak{g} \otimes t \mathbb{C}[t, u]$ acts trivially. Recall that we get a highly nontrivial action of $\mathfrak{g}[t, u]$ when we consider fusion products of these modules with respect to the variable $t$. The bigraded fusion product

$$
\overline{\mathrm{D}^{u}\left(\ell, \ell \lambda^{1}\right)} * \cdots * \overline{\mathrm{D}^{u}\left(\ell, \ell \lambda^{1}\right)} * \overline{\mathrm{D}^{u}\left(\ell, \ell \lambda^{1}+\lambda^{0}\right)}
$$

is a cyclic module for the Lie algebra $\boldsymbol{U}\left(\mathfrak{g} \otimes \mathbb{C}[t, u] /\left\langle t^{N}, u^{\lambda^{1}\left(\theta^{\vee}\right)+1}\right\rangle\right)$. Note the similarity but also the difference between (3-5) and (3-6). In (3-5) we consider the fusion product (with respect to the variable $t$ ) of $\mathfrak{g}[t]$-modules. The $\mathfrak{g}[t, u]$-module structure comes into the picture only by the filtration defined in Section 3C. We would like to remind the reader that if $\lambda^{0} \neq 0$, then $\mathfrak{g}$ is of classical type or $\mathrm{G}_{2}$. 
Theorem 3.5. Let $\lambda^{1} \in L^{+}$and $\lambda^{0} \in P^{+}$such that $\lambda^{0}\left(\theta^{\vee}\right) \leq \ell$. We have an isomorphism of $\boldsymbol{U}\left(\mathfrak{g} \otimes \mathbb{C}[t, u] /\left\langle t^{N}, u^{\lambda^{1}\left(\theta^{\vee}\right)+1}\right\rangle\right)$-modules

$$
\operatorname{gr}_{t^{N}} \mathrm{~T}(\ell, N) \cong \overline{\mathrm{D}^{u}\left(\ell, \ell \lambda^{1}\right)} * \cdots * \overline{\mathrm{D}^{u}\left(\ell, \ell \lambda^{1}\right)} * \overline{\mathrm{D}^{u}\left(\ell, \ell \lambda^{1}+\lambda^{0}\right)} .
$$

Proof. Let $v_{\ell}^{*(N-1)} * v_{0}$ be the highest weight vector of the right-hand side. The isomorphism between $\operatorname{gr}_{t^{N}} \mathrm{~T}(N)$ and $\boldsymbol{U}\left(\mathfrak{g} \otimes \mathbb{C}[t, u] /\left\langle t^{N}, u^{\lambda^{1}\left(\theta^{\vee}\right)+1}\right\rangle\right)$ (see Lemma 3.4) induces a natural surjective map

$$
\mathrm{gr}_{t^{N}} \mathrm{~T}(N) \rightarrow \boldsymbol{U}\left(\mathfrak{g} \otimes \mathbb{C}[t, u] /\left\langle t^{N}, u^{\lambda^{1}\left(\theta^{\vee}\right)+1}\right\rangle\right) \circ\left(v_{\ell}^{*(N-1)} * v_{0}\right) .
$$

It remains to prove that this map induces an isomorphism between the cyclic module $\operatorname{gr}_{t^{N}} \mathrm{~T}(\ell, N)$ and the fusion product. Since the dimensions of the modules coincide it is enough to show that all relations which hold in $\operatorname{gr}_{t^{N}} \mathrm{~T}(\ell, N)$ also hold on the right-hand side.

Recall that a presentation of $\operatorname{gr}_{t^{N}} \mathrm{~T}(\ell, N)$ is given by two types of relations, the ones coming from the presentation of the Demazure module and the ones coming from going to the associated graded space with respect to the $t^{N}$-filtration. We start by proving that the defining relations of $\mathrm{D}\left(\ell, \ell N \lambda^{1}+\lambda^{0}\right)$ given for $v$ in Proposition 3.1 are satisfied by $v_{\ell} * \cdots * v_{\ell} * v_{0}$. Since the relations (3-2) and the first part of (3-3) are obviously satisfied it remains to verify the second part of (3-3) and (3-4). Write $\left(\ell N \lambda^{1}+\lambda^{0}\right)\left(\beta^{\vee}\right)$ as in (3-1). We start by proving that

$$
\left(x_{-\beta} \otimes u^{j_{\beta}} t^{r_{\beta}}\right)\left(v_{\ell}^{*(N-1)} * v_{0}\right)=0, \quad \text { where } p_{\beta}=j_{\beta} N+r_{\beta}, 0 \leq r_{\beta}<N .
$$

Since $\lambda^{0}\left(\beta^{\vee}\right) \leq d_{\beta} \ell$, we have

$$
p_{\beta}= \begin{cases}N \lambda^{1}\left(\beta^{\vee}\right) d_{\beta}^{-1} & \text { if } \lambda^{0}\left(\beta^{\vee}\right)=0, \\ N \lambda^{1}\left(\beta^{\vee}\right) d_{\beta}^{-1}+1 & \text { else. }\end{cases}
$$

In either case $j_{\beta} \geq \lambda^{1}\left(\beta^{\vee}\right) d_{\beta}^{-1}$ and thus $\left(x_{-\beta} \otimes u^{j_{\beta}} t^{r_{\beta}}\right) v_{\ell}=0$ follows immediately from the defining relations of $\mathrm{D}\left(\ell, \ell \lambda^{1}\right)$. If $r_{\beta} \neq 0$ we can replace $t^{r_{\beta}}$ by $\left(t-z_{N}\right)^{r_{\beta}}$ in the associated graded space and obtain that the element in (3-7) acts trivially on $v_{0}$. If $r_{\beta}=0$, then $p_{\beta}$ is divisible by $N$, which forces $\lambda^{0}\left(\beta^{\vee}\right)=0$. Therefore, in this case we obtain $j_{\beta}=\lambda^{1}\left(\beta^{\vee}\right) d_{\beta}^{-1}$, and $\left(x_{-\beta} \otimes u^{j_{\beta}}\right) v_{0}=0$ follows immediately from the defining relations of $\mathrm{D}\left(\ell, \ell \lambda^{1}+\lambda^{0}\right)$. It remains to consider the relations (3-4). So suppose we have

$$
p_{\beta}-1=N \frac{\lambda^{1}\left(\beta^{\vee}\right)}{d_{\beta}}+\frac{\lambda^{0}\left(\beta^{\vee}\right)-m_{\beta}}{d_{\beta} \ell}=j_{\beta} N+r_{\beta}, \quad 0 \leq r_{\beta}<N .
$$

Since $m_{\beta}<d_{\beta} \ell$, we must have $m_{\beta}=\lambda^{0}\left(\beta^{\vee}\right) \neq 0$ and hence $p_{\beta}-1=N \lambda^{1}\left(\beta^{\vee}\right) d_{\beta}^{-1}$. It follows that $j_{\beta}=\lambda^{1}\left(\beta^{\vee}\right) d_{\beta}^{-1}$ and therefore $\left(x_{-\beta} \otimes u^{j_{\beta}}\right) v_{\ell}=0$. So we have

$$
\left(x_{-\beta} \otimes u^{j_{\beta}}\right)^{m_{\beta}+1}\left(v_{\ell}^{*(N-1)} * v_{0}\right)=v_{\ell}^{*(N-1)} *\left(x_{-\beta} \otimes u^{j_{\beta}}\right)^{m_{\beta}+1} v_{0}=0 .
$$


We now consider the relations coming from the $t^{N}$-filtration. Suppose

$$
\begin{aligned}
& M=\sum_{m} \sum_{\substack{i_{1}, \ldots, i_{m} \\
j_{1}, \ldots, j_{m}}} k(m)_{\substack{i_{1}, \ldots, i_{m} \\
j_{1} \ldots, j_{m}}}\left(x_{i_{1}} \otimes t^{i_{1} N+j_{1}}\right) \cdots\left(x_{i_{m}} \otimes t^{i_{m} N+j_{m}}\right) \\
& \in \boldsymbol{U}\left(\mathfrak{g} \otimes \mathbb{C}[t] / t^{\left(\lambda\left(\theta^{\vee}\right)+1\right) N}\right)
\end{aligned}
$$

is a linear combination of monomials with fixed $t^{N}$-degree such that $w=M v \neq 0$ in $\mathrm{D}\left(\ell, \ell N \lambda^{1}+\lambda^{0}\right)$ but the image $\bar{w}=0$ in $\operatorname{gr}_{t^{N}} \mathrm{~T}(\ell, N)$. This is only possible if there exists a linear combination of monomials of greater $t^{N}$-degree

$$
\begin{aligned}
M^{\prime}=\sum_{m^{\prime}} \sum_{\substack{p_{1}, \ldots, p_{m^{\prime}} \\
q_{1} \ldots, q_{m^{\prime}}}} k\left(m^{\prime}\right) p_{\substack{1 \\
q_{1}, \ldots, q_{m^{\prime}}}}\left(x_{p_{1}} \otimes t^{p_{1} N+q_{1}}\right) \cdots\left(x_{p_{m^{\prime}}} \otimes t^{p_{m^{\prime}} N+q_{m^{\prime}}}\right) \\
\in \boldsymbol{U}\left(\mathfrak{g} \otimes \mathbb{C}[t] / t^{\left(\lambda\left(\theta^{\vee}\right)+1\right) N}\right)
\end{aligned}
$$

such that $w=M v=M^{\prime} v$ in $\mathrm{D}\left(\ell, \ell N \lambda^{1}+\lambda^{0}\right)$. We assume in what follows that $M^{\prime}$ is of maximal $t^{N}$-degree. We have $\left(M-M^{\prime}\right) v=0$, so the difference $M-M^{\prime}$ is an element in the left ideal generated by the elements in (3-2)-(3-4). Since $M^{\prime}$ is of higher $t^{N}$-degree we get $\overline{M-M^{\prime}}=\bar{M}$ in $\operatorname{gr}_{t^{N}} \mathrm{~T}(N)$, and since all defining relations of $\mathrm{D}\left(\ell, \ell N \lambda^{1}+\lambda^{0}\right)$ are satisfied by $v_{\ell}^{*(N-1)} * v_{0}$ we get $\Psi(\bar{M}) \circ\left(v_{\ell}^{*(N-1)} * v_{0}\right)=0$, which shows that the natural surjective map

$$
\operatorname{gr}_{t^{N}} \mathrm{~T}(N) \rightarrow \boldsymbol{U}\left(\mathfrak{g} \otimes \mathbb{C}[t, u] /\left\langle t^{N}, u^{\lambda^{1}\left(\theta^{\vee}\right)+1}\right\rangle\right) \circ\left(v_{\ell}^{*(N-1)} * v_{0}\right)
$$

induces an isomorphism of cyclic modules $\operatorname{gr}_{t^{N}} \mathrm{~T}(\ell, N) \cong \overline{\mathrm{D}^{u}\left(\ell, \ell \lambda^{1}\right)} * \cdots *$ $\overline{\mathrm{D}^{u}\left(\ell, \ell \lambda^{1}\right)} * \overline{\mathrm{D}^{u}\left(\ell, \ell \lambda^{1}+\lambda^{0}\right)}$.

For the rest of this section we discuss a crucial consequence of our result.

Corollary 3.6. Let $\ell \in \mathbb{N}, \lambda^{1} \in L^{+}$and $\lambda^{0} \in P^{+}$such that $\lambda^{0}\left(\theta^{\vee}\right) \leq \ell$.

(1) The fusion product $\overline{\mathrm{D}^{u}\left(\ell, \ell \lambda^{1}\right)} * \cdots * \overline{\mathrm{D}^{u}\left(\ell, \ell \lambda^{1}\right)} * \overline{\mathrm{D}^{u}\left(\ell, \ell \lambda^{1}+\lambda^{0}\right)}$ is independent of the fusion parameters.

(2) The fusion product $\mathrm{V}\left(\ell \lambda^{1}\right)^{*(N-1)} * \mathrm{~V}\left(\ell \lambda^{1}+\lambda^{0}\right)$ is independent of the fusion parameters.

(3) We have an isomorphism of $\boldsymbol{U}\left(\mathfrak{g} \otimes \mathbb{C}[t] / t^{N}\right)$-modules

$\mathrm{V}\left(\ell \lambda^{1}\right)^{*(N-1)} * \mathrm{~V}\left(\ell \lambda^{1}+\lambda^{0}\right) \cong \mathrm{D}\left(\ell, \ell N \lambda^{1}+\lambda^{0}\right) /\left(\mathfrak{g} \otimes t^{N} \mathbb{C}[t]\right) \mathrm{D}\left(\ell, \ell N \lambda^{1}+\lambda^{0}\right)$.

(4) If $\lambda^{0}\left(\theta^{\vee}\right) \leq 1$, the truncated level one Demazure module is isomorphic to the truncated level $\ell$ Demazure module

$$
\begin{aligned}
\mathrm{D}\left(1, \ell N \lambda^{1}+\lambda^{0}\right) /\left(\mathfrak{g} \otimes t^{N} \mathbb{C}[t]\right) & \mathrm{D}\left(1, \ell N \lambda^{1}+\lambda^{0}\right) \\
& \cong \mathrm{D}\left(\ell, \ell N \lambda^{1}+\lambda^{0}\right) /\left(\mathfrak{g} \otimes t^{N} \mathbb{C}[t]\right) \mathrm{D}\left(\ell, \ell N \lambda^{1}+\lambda^{0}\right) .
\end{aligned}
$$

Proof. Since the fusion product $\mathrm{V}\left(\ell \lambda^{1}\right)^{*(N-1)} * V\left(\ell \lambda^{1}+\lambda^{0}\right)$ is isomorphic to the zeroth graded component of $\overline{\mathrm{D}^{u}\left(\ell, \ell \lambda^{1}\right)} * \cdots * \overline{\mathrm{D}^{u}\left(\ell, \ell \lambda^{1}\right)} * \overline{\mathrm{D}^{u}\left(\ell, \ell \lambda^{1}+\lambda^{0}\right)}$ (with respect to the $u$-grading) the statement follows from Theorem 3.5 . 
Remark. Theorem 3.5 generalizes a result of [Feigin 2008], where the theorem was proved for $\ell=1, \lambda^{0}=0$ and $\lambda^{1}=\theta$. Unfortunately, the proof in that paper has a gap (personal communication by the author), which is now fixed by the proof above. Ravinder [2014] used the result of [Feigin 2008] to prove a presentation for the fusion product $\mathrm{V}(\theta)^{* N} * \mathrm{D}(1, \theta)^{* M}$.

\section{Truncated Weyl modules and PBW type basis}

In this section we give some evidence for the conjecture made by Chari, Fourier and Sagaki on truncated Weyl modules (see [Chari et al. 2014a; Fourier 2015]). For the reader's convenience we state the precise conjecture in this paper (Conjecture 4.1). Finally, we consider the case $\mathfrak{g}=\mathfrak{s l}_{2}$ and compute a PBW type basis.

4A. Let $P^{+}(\lambda, N)$ be the set of $N$-tuples of dominant integral weights $\lambda=$ $\left(\lambda_{1}, \ldots, \lambda_{N}\right)$ such that $\sum_{i} \lambda_{i}=\lambda$. Let $\lambda=\left(\lambda_{1}, \ldots, \lambda_{N}\right), \boldsymbol{\mu}=\left(\mu_{1}, \ldots, \mu_{N}\right) \in$ $P^{+}(\lambda, N)$. For a positive root $\beta$ define

$$
r_{\beta, k}(\lambda)=\min \left\{\left(\lambda_{i_{1}}+\cdots+\lambda_{i_{k}}\right)\left(\beta^{\vee}\right) \mid 1 \leq i_{1}<\cdots<i_{k} \leq N\right\} .
$$

We say $\lambda \preceq \mu$ if

$$
r_{\beta, k}(\lambda) \leq r_{\beta, k}(\boldsymbol{\mu}) \text { for all } \beta \in R^{+} \text {and } 1 \leq k \leq N .
$$

The above partial order was considered by Chari et al. [2014a], who observed that for a tuple $\lambda$ the dimension of the tensor product of the corresponding finite-dimensional simple $\mathfrak{g}$-modules increases along $\preceq$. Moreover, they proved in certain cases (for instance when $\lambda$ is a multiple of a fundamental minuscule weight) that there exists an inclusion of tensor products along with the partial order and conjectured that this remains true for $N=2$ and arbitrary $\lambda$ (see [Chari et al. 2014a, Conjecture 2.3]). Using the unique maximal element in the partially ordered set $P^{+}(\lambda, N)$ one can formulate a conjecture on truncated Weyl modules, which we will explain now.

Definition. Let $\lambda \in P^{+}$. The truncated Weyl module $\mathrm{W}(\lambda, N)$ is a cyclic module for $\boldsymbol{U}\left(\mathfrak{g} \otimes \mathbb{C}[t] / t^{N}\right)$ generated by $w_{\lambda, N}$ with relations

$$
\begin{gathered}
\left(\mathfrak{n}^{+} \otimes \mathbb{C}[t] / t^{N}\right) w_{\lambda, N}=0, \quad\left(h \otimes t^{s}\right) w_{\lambda, N}=\delta_{s, 0} \lambda(h) w_{\lambda, N} \forall h \in \mathfrak{h}, s \geq 0, \\
\left(x_{-\beta} \otimes 1\right)^{\lambda\left(\beta^{\vee}\right)+1} w_{\lambda, N}=0 \quad \forall \beta \in R^{+} .
\end{gathered}
$$

The following conjecture gives a connection between truncated Weyl modules and fusion products of irreducible finite-dimensional $\mathfrak{g}$-modules.

Conjecture 4.1. Let $\lambda \in P^{+}$such that $|\lambda| \geq N$, and let $\lambda=\left(\lambda_{1}, \ldots, \lambda_{N}\right)$ be the unique maximal element in $P^{+}(\lambda, N)$. Then we have an isomorphism of $\boldsymbol{U}\left(\mathfrak{g} \otimes \mathbb{C}[t] / t^{N}\right)$-modules

$$
\mathrm{W}(\lambda, N) \cong V\left(\lambda_{1}\right) * \cdots * V\left(\lambda_{N}\right) .
$$


The following result proves the above conjecture for certain classes of dominant integral weights.

Theorem 4.2. Let $\lambda \in L^{+}$and $\lambda^{0} \in P^{+}$such that $\lambda^{0}\left(\theta^{\vee}\right) \leq 1$ and $\left|N \lambda+\lambda^{0}\right| \geq N$. Then we have an isomorphism of $\boldsymbol{U}\left(\mathfrak{g} \otimes \mathbb{C}[t] / t^{N}\right)$-modules

$$
\mathrm{W}\left(N \lambda+\lambda^{0}, N\right) \cong V(\lambda) * \cdots * V(\lambda) * V\left(\lambda+\lambda^{0}\right) .
$$

Proof. If $\lambda=0$, there is nothing to prove. By Corollary 3.6 we obtain that

$$
V(\lambda) * \cdots * V(\lambda) * V\left(\lambda+\lambda^{0}\right) \cong \mathrm{D}\left(1, N \lambda+\lambda^{0}\right) /\left(\mathfrak{g} \otimes t^{N} \mathbb{C}[t]\right) \mathrm{D}\left(1, N \lambda+\lambda^{0}\right) .
$$

We show that the defining relations of $\mathrm{D}\left(1, N \lambda+\lambda^{0}\right) /\left(\mathfrak{g} \otimes t^{N} \mathbb{C}[t]\right) \mathrm{D}\left(1, N \lambda+\lambda^{0}\right)$ hold in the truncated Weyl module. We shall prove only the nonobvious relations. Let $\beta \in R^{+}$and write $\left(N \lambda+\lambda^{0}\right)\left(\beta^{\vee}\right)$ as in (3-1). Then, as before,

$$
p_{\beta}-1= \begin{cases}N \lambda\left(\beta^{\vee}\right) d_{\beta}^{-1} & \text { if } \lambda^{0}\left(\beta^{\vee}\right) \neq 0, \\ N \lambda\left(\beta^{\vee}\right) d_{\beta}^{-1}-1 & \text { else. }\end{cases}
$$

We consider four cases. If $\lambda\left(\beta^{\vee}\right) \neq 0$ and $\lambda^{0}\left(\beta^{\vee}\right) \neq 0$, then $p_{\beta} \geq p_{\beta}-1 \geq N$ and hence

$$
\left(x_{-\beta} \otimes t^{p_{\beta}}\right) w_{N \lambda+\lambda^{0}, N}=\left(x_{-\beta} \otimes t^{p_{\beta}-1}\right) w_{N \lambda+\lambda^{0}, N}=0 .
$$

If $\lambda\left(\beta^{\vee}\right) \neq 0$ and $\lambda^{0}\left(\beta^{\vee}\right)=0$, then $p_{\beta} \geq N$ and $m_{\beta}=d_{\beta}$ (recall that (3-4) was only considered when $\left.m_{\beta}<d_{\beta}\right)$. If $\lambda\left(\beta^{\vee}\right)=0$ and $\lambda^{0}\left(\beta^{\vee}\right)=0$, there is nothing to show; so consider the last case, $\lambda\left(\beta^{\vee}\right)=0$ and $\lambda^{0}\left(\beta^{\vee}\right) \neq 0$. In this case $p_{\beta}=1$ and $m_{\beta}=\lambda^{0}\left(\beta^{\vee}\right)$. Thus we have to prove

$$
\left(x_{-\beta} \otimes t\right) w_{N \lambda+\lambda^{0}, N}=\left(x_{-\beta} \otimes 1\right)^{m_{\beta}+1} w_{N \lambda+\lambda^{0}, N}=0,
$$

where the last equality is clear. Note that it is enough to prove that $\left(x_{-\beta} \otimes t\right)$ acts by zero on the highest weight vector of the local Weyl module $\mathrm{W}_{\text {loc }}\left(N \lambda+\lambda^{0}\right)$. Since $\mathrm{W}_{\text {loc }}\left(N \lambda+\lambda^{0}\right) \cong \mathrm{W}_{\text {loc }}^{z_{1}}(N \lambda) * \mathrm{~W}_{\text {loc }}^{z_{2}}\left(\lambda^{0}\right)$ we get

$$
\left(x_{-\beta} \otimes t\right)\left(w_{N \lambda} * w_{\lambda^{0}}\right)=\left(x_{-\beta} \otimes\left(t-z_{2}\right)\right)\left(w_{N \lambda} * w_{\lambda^{0}}\right)=w_{N \lambda} *\left(x_{-\beta} \otimes t\right) w_{\lambda^{0}} .
$$

If $\mathfrak{g}$ is not of type $\mathrm{G}_{2}$, then $\mathrm{W}_{\text {loc }}\left(\lambda^{0}\right)$ is irreducible and the statement follows. If $\mathfrak{g}$ is $\mathrm{G}_{2}$ it is easy to see that the only positive $\operatorname{root} \beta$ with $\left(x_{-\beta} \otimes t\right) w_{\lambda^{0}} \neq 0$ is the longest short root $\beta=\alpha_{1}+2 \alpha_{2}$. But then $\lambda\left(\beta^{\vee}\right) \neq 0$.

We shall show that $\left(\lambda, \ldots, \lambda, \lambda+\lambda^{0}\right)$ is in fact the unique maximal element in $P^{+}\left(N \lambda+\lambda^{0}, N\right)$. Since $\lambda^{0}\left(\theta^{\vee}\right) \leq 1$, there exists at most one simple root $\alpha$ such that $\lambda^{0}\left(\alpha^{\vee}\right)>0$. Without loss of generality we suppose $\lambda^{0}\left(\alpha_{j}^{\vee}\right)=0$ for all $j>1$. Assume that $\left(\mu_{1}, \ldots, \mu_{N}\right) \in P^{+}\left(N \lambda+\lambda^{0}, N\right)$ such that $\left(\lambda, \ldots, \lambda, \lambda+\lambda^{0}\right) \preceq\left(\mu_{1}, \ldots, \mu_{N}\right)$. We fix a simple root $\alpha_{j}$ and a permutation $\sigma_{j}$ such that

$$
\mu_{\sigma_{j}(1)}\left(\alpha_{j}^{\vee}\right) \leq \cdots \leq \mu_{\sigma_{j}(N)}\left(\alpha_{j}^{\vee}\right)
$$


We write $\mu_{\sigma_{j}(i)}\left(\alpha_{j}^{\vee}\right)=\lambda\left(\alpha_{j}^{\vee}\right)+\epsilon_{i}(j)+\delta_{i, N} \lambda^{0}\left(\alpha_{j}^{\vee}\right)$ for integers $\epsilon_{i}(j)$. By our assumptions we obtain

$$
0 \leq \epsilon_{1}(j) \leq \cdots \leq \epsilon_{N-1}(j) \leq \epsilon_{N}(j)+\lambda^{0}\left(\alpha_{j}^{\vee}\right) \quad \text { and } \quad \sum_{p=1}^{N} \epsilon_{p}(j)=0 .
$$

Hence, up to a permutation we have $\mu_{i}=\lambda$ for $1 \leq i \leq N-1$ and $\mu_{N}=\lambda+\lambda^{0}$.

4B. For the rest of this section we prove the conjecture for $\mathfrak{s l}_{2}$ and compute a PBW type basis. For $0 \leq j<N$, let $S\left(k^{N-j},(k+1)^{j}\right)$ be the set of tuples $\left(i_{0}, \ldots, i_{N-1}\right)$ satisfying

$$
\sum_{p=0}^{N-1} \frac{N !}{N-p} i_{p} \leq N ! k-\sum_{\ell=0}^{N-4} \frac{N !}{(N-\ell) !}(N-\ell-2) ! b_{\ell}+j(N-1) !
$$

for integers $b_{\ell}$ defined as follows: $0 \leq b_{\ell}<N-\ell$ and

$$
\begin{aligned}
& i_{0}-j=b_{0} \bmod N, \\
& i_{\ell}+\left(b_{\ell-1} \bmod N-\ell\right)=b_{\ell} \bmod N-\ell \text { for } \ell=1, \ldots, N-4 .
\end{aligned}
$$

The theorem we shall prove is the following.

Theorem 4.3. Let $m \in \mathbb{Z}_{+}$and write $m=k N+j$ for $0 \leq j<N$.

(1) We have an isomorphism of $\boldsymbol{U}\left(\mathfrak{s l}_{2} \otimes \mathbb{C}[t] / t^{N}\right)$-modules

$$
\mathrm{W}(m, N) \cong V(k)^{*(N-j)} * V(k+1)^{* j} .
$$

(2) A PBW type basis of $\mathrm{W}(m, N)$ is given by

$$
\left\{\left(x_{-\alpha} \otimes 1\right)^{i_{0}} \cdots\left(x_{-\alpha} \otimes t^{N-1}\right)^{i_{N-1}} w_{m, N} \mid\left(i_{0}, \ldots, i_{N-1}\right) \in S\left(k^{N-j},(k+1)^{j}\right)\right\} .
$$

A simple calculation similar to the one above shows that $(k, \ldots, k, k+1, \ldots, k+1)$ $\in P^{+}(m, N)$ is in fact the unique maximal element.

The rest of this section is dedicated to the proof of Theorem 4.3.

4C. We start by proving the first part of the theorem. A presentation of the fusion product as a $\boldsymbol{U}\left(\mathfrak{s l}_{2} \otimes \mathbb{C}[t]\right)$ was given in [Chari and Venkatesh 2015]. So by their results it is enough to show that the highest weight vector of $\mathrm{W}(m, N)$ satisfies the defining relations of $V(k)^{*(N-j)} * V(k+1)^{* j}$ given in [Chari and Venkatesh 2015, Proposition 2.7], which are

$$
\begin{aligned}
\boldsymbol{x}_{-\alpha}(r, s)=\sum_{\left(b_{p}\right)_{p \geq 0} \in \boldsymbol{S}(r, s)}\left(x_{-\alpha} \otimes 1\right)^{b_{0}}\left(x_{-\alpha} \otimes t\right)^{b_{1}} \cdots\left(x_{-\alpha} \otimes t^{s}\right)^{b_{s}}, \\
s, r, \ell \in \mathbb{N}, r+s \geq 1+r \ell+q+p,
\end{aligned}
$$


where $q=\max \{0,(N-\ell) k\}, p=\max \{0, j-\ell\}$ and $\boldsymbol{S}(r, s)$ is the set of tuples $\left(b_{p}\right)_{p \geq 0}$ satisfying $b_{0}+\cdots+b_{s}=r$ and $b_{1}+2 b_{2}+\cdots+s b_{s}=s$. We assume that $r+s \leq m$, because otherwise the claim follows from the following result of Garland [1978]:

$$
\left(x_{\alpha} \otimes t\right)^{(s)}\left(x_{-\alpha} \otimes 1\right)^{(s+r)}-(-1)^{s} \boldsymbol{x}_{-\alpha}(r, s) \in \boldsymbol{U}(\mathfrak{g}[t]) \mathfrak{n}^{+}[t] .
$$

Our aim is to prove that for any tuple $\left(b_{p}\right)_{p \geq 0} \in S(r, s)$ there exists $p \geq N$ such that $b_{p} \neq 0$. Assume this is not the case. If $\ell \geq N$ we obtain

$$
r N \geq r+s \geq 1+r \ell \geq 1+r N
$$

which is obviously a contradiction. So assume $l \leq N-1$. It follows that

$$
m \geq r+s \geq 1+r \ell+(N-\ell) k+p=1+\ell(r-k)+m-j+p
$$

and thus $r \leq k$. Therefore we obtain the contradiction

$$
1+\ell(r-k)+m-j+p \leq r+s \leq r N \Rightarrow 1 \leq(N-\ell)(r-k)-p .
$$

Hence

$$
\mathrm{W}(m, N) \cong V(k)^{*(N-j)} * V(k+1)^{* j} .
$$

4D. Now we will prove the second part of the theorem. For simplicity we write $f_{i}$ for $x_{-\alpha} \otimes t^{i}, 1 \leq i \leq N-1$, and consider the map sh : $\boldsymbol{U}\left(\mathfrak{n}^{-}[t]\right) \rightarrow \boldsymbol{U}\left(\mathfrak{n}^{-}[t]\right)$ given by $\operatorname{sh}\left(f_{i}\right)=f_{i+1}$. We will need the following result from [Feigin and Feigin 2002].

Proposition 4.4. Let $k_{1} \leq k_{2} \leq \cdots \leq k_{N}$. We have a short exact sequence of $\boldsymbol{U}\left(\mathfrak{n}^{-}[t]\right)$-modules

$0 \rightarrow V\left(k_{1}\right) * \cdots * V\left(k_{N-1}\right) \stackrel{\mathrm{sh}}{\longrightarrow} V\left(k_{1}\right) * \cdots * V\left(k_{N}\right) \stackrel{f_{0}^{-1}}{\longrightarrow} V\left(k_{1}\right) * \cdots * V\left(k_{N}-1\right) \rightarrow 0$.

Using this proposition one can construct inductively a PBW type basis of the fusion product. To be more precise, we have

$$
B\left(k_{1}, \ldots, k_{N}\right)=B\left(k_{1}, \ldots, k_{N-1}\right)_{\mathrm{sh}} \cup f_{0} B\left(k_{1}, \ldots, k_{N}-1\right),
$$

where $B(\cdot)$ denotes a basis of the appropriate fusion product.

Example. We have $B(1,2)=B(1)_{\mathrm{sh}} \cup f_{0} B(1,1)$ and hence

$$
B(1,2)=\left\{1, f_{0}\right\}_{\mathrm{sh}} \cup f_{0}\left\{1, f_{0}, f_{0}^{2}, f_{1}\right\}=\left\{1, f_{1}, f_{0} f_{1}, f_{0}, f_{0}^{2}, f_{0}^{3}\right\} .
$$

Lemma 4.5. We have the recursion formula

$$
B\left(k^{N}\right)=\bigcup_{r=0}^{k} f_{0}^{N r} B\left((k-r)^{N-1}\right)_{\mathrm{sh}} \cup \bigcup_{j=1}^{N-1} \bigcup_{r=1}^{k} f_{0}^{N r-j} B\left((k-r)^{N-j},(k-r+1)^{j-1}\right)_{\mathrm{sh}} .
$$


Proof. The proof follows by repeated applications of (4-4); for the convenience of the reader we present the first step:

$$
\begin{aligned}
B\left(k^{N}\right) & =B\left(k^{N-1}\right)_{\mathrm{sh}} \cup f_{0} B\left((k-1)^{1}, k^{N-1}\right) \\
& =B\left(k^{N-1}\right)_{\mathrm{sh}} \cup f_{0} B\left((k-1)^{1}, k^{n-2}\right)_{\mathrm{sh}} \cup f_{0}^{2} B\left((k-1)^{2}, k^{N-2}\right) \\
& =\cdots=\bigcup_{r=0}^{N-1} f_{0}^{r} B\left((k-1)^{r}, k^{N-1-r}\right)_{\mathrm{sh}} \cup f_{0}^{N} B\left((k-1)^{N}\right) .
\end{aligned}
$$

The formula now follows by proceeding in the same way with $B\left((k-1)^{N}\right)$.

Theorem 4.6. A PBW type basis of the truncated Weyl module $\mathrm{W}(k N, N)$ is given by

$$
B\left(k^{N}\right)=\left\{f_{0}^{i_{0}} f_{1}^{i_{1}} \ldots f_{N-1}^{i_{N-1}} \mid\left(i_{0}, \ldots, i_{N-1}\right) \in S\left(k^{N}\right)\right\} .
$$

Example. (1) For $N=1$ we get that $S(k)$ is the set of 1-tuples $\left(i_{0}\right)$ satisfying

$$
i_{0}=\sum_{j=0}^{0} \frac{1 !}{1-j} i_{j} \leq 1 ! k-\sum_{\ell=0}^{-3} \frac{1 !}{(1-\ell) !}(1-\ell-2) ! b_{\ell}=k,
$$

$$
\text { so } S(k)=\{0,1, \ldots, k\} \text { and } B(k)=\left\{f_{0}^{j} \mid j=0, \ldots, k\right\} \text {. }
$$

(2) For $N=4$ and $k=2$ we get that $S\left(2^{4}\right)$ is the set of quadruples $\left(i_{0}, i_{1}, i_{2}, i_{3}\right)$ satisfying

$$
6 i_{0}+8 i_{1}+12 i_{2}+24 i_{3} \leq 48-2 b_{0},
$$

where $i_{0}=b_{0} \bmod 4$ and

$$
B\left(2^{4}\right)=\left\{f_{0}^{i_{0}} f_{1}^{i_{1}} f_{2}^{i_{2}} f_{3}^{i_{3}} \mid\left(i_{0}, i_{1}, i_{2}, i_{3}\right) \in S\left(2^{4}\right)\right\} .
$$

Proof. The proof of Theorem 4.6 proceeds by upward induction on $N$. The initial step is obvious (see also the previous example) and the induction begins. So suppose that the theorem holds for all integers less than $N$.

Claim. For all $M<N$ we have

$$
B\left(k^{M-j},(k+1)^{j}\right)=\left\{f_{0}^{i_{0}} f_{1}^{i_{1}} \cdots f_{M-1}^{i_{M-1}} \mid\left(i_{0}, \ldots, i_{M-1}\right) \in S\left(k^{M-j},(k+1)^{j}\right)\right\} .
$$

Proof of the claim. We use induction. There is nothing to prove if $j=0$. Assuming $j>0$, we obtain

$$
\begin{aligned}
B\left(k^{M-j},(k+1)^{j}\right) & \\
= & B\left(k^{M-j},(k+1)^{j-1}\right)_{\mathrm{sh}} \cup f_{0} B\left(k^{M-j+1},(k+1)^{j-1}\right) \\
= & \left\{f_{0}^{i_{0}} f_{1}^{i_{1}} \cdots f_{M-2}^{i_{M-2}} \mid\left(i_{0}, \ldots, i_{M-2}\right) \in S\left(k^{M-j},(k+1)^{j-1}\right)\right\}_{\mathrm{sh}} \\
& \quad \cup f_{0}\left\{f_{0}^{i_{0}} f_{1}^{i_{1}} \cdots f_{M-1}^{i_{M-1}} \mid\left(i_{0}, \ldots, i_{M-1}\right) \in S\left(k^{M-j+1},(k+1)^{j-1}\right)\right\} .
\end{aligned}
$$


The shift by the map sh leads to the following description:

$$
\begin{aligned}
& \left\{f_{0}^{i_{0}} f_{1}^{i_{1}} \cdots f_{M-2}^{i_{M-2}} \mid\left(i_{0}, \ldots, i_{M-2}\right) \in S\left(k^{M-j},(k+1)^{j-1}\right)\right\}_{\mathrm{sh}} \\
& =\left\{f_{1}^{i_{1}} f_{2}^{i_{2}} \cdots f_{M-1}^{i_{M-1}} \mid\right. \\
& \left.\quad \sum_{p=1}^{M-1} \frac{M !}{M-p} i_{p} \leq M ! k-\sum_{\ell=1}^{M-4} \frac{M !}{(M-\ell) !}(M-\ell-2) ! b_{\ell}+M(j-1)(M-2) !\right\}
\end{aligned}
$$

with

$$
\begin{aligned}
& i_{1}-j+1=b_{1} \bmod M-1, \\
& i_{\ell}+\left(b_{\ell-1} \bmod M-\ell\right)=b_{\ell} \bmod M-\ell \text { for } \ell=2, \ldots, M-4,
\end{aligned}
$$

and

$$
\begin{aligned}
& f_{0}\left\{f_{0}^{i_{0}} f_{1}^{i_{1}} \cdots f_{M-1}^{i_{M-1}} \mid\left(i_{0}, \ldots, i_{M-1}\right) \in S\left(k^{M-j+1},(k+1)^{j-1}\right)\right\} \\
& \quad=\left\{f_{0}^{i_{0}+1} f_{1}^{i_{1}} \cdots f_{M-1}^{i_{M-1}} \mid\right. \\
& \left.\quad \sum_{p=0}^{M-1} \frac{M !}{M-p} i_{p} \leq M ! k-\sum_{\ell=0}^{M-4} \frac{M !}{(M-\ell) !}(M-\ell-2) ! b_{\ell}+(j-1)(M-1) !\right\} \\
& \text { with } \quad\left\{f_{0}^{i_{0}} f_{1}^{i_{1}} \cdots f_{M-1}^{i_{M-1}} \mid\right.
\end{aligned}
$$

$$
\begin{aligned}
& i_{0}-j=b_{0} \bmod M, \\
& i_{\ell}+\left(b_{\ell-1} \bmod M-\ell\right)=b_{\ell} \bmod M-\ell \quad \text { for } \ell=1, \ldots, M-4 .
\end{aligned}
$$

Therefore, the claim follows with

$$
\begin{aligned}
& \left\{f_{0}^{i_{0}} f_{1}^{i_{1}} \cdots f_{M-2}^{i_{M-2}} \mid\left(i_{0}, \ldots, i_{M-2}\right) \in S\left(k^{M-j},(k+1)^{j-1}\right)\right\}_{\mathrm{sh}} \\
& =\left\{f_{0}^{0} f_{1}^{i_{1}} \cdots f_{M-1}^{i_{M-1}} \mid\right. \\
& \left.\quad \sum_{p=0}^{M-1} \frac{M !}{M-p} i_{p} \leq M ! k-\sum_{\ell=0}^{M-4} \frac{M !}{(M-\ell) !}(M-\ell-2) ! b_{\ell}+j(M-1) !\right\} .
\end{aligned}
$$

Now it is easy to verify with Lemma 4.5 that the theorem holds.

The proof of Theorem 4.6 gives the following.

Corollary 4.7. A PBW type basis of the truncated Weyl module $\mathrm{W}(k N+j, N)$ is given by

$$
\left\{f_{0}^{i_{0}} f_{1}^{i_{1}} \ldots f_{N-1}^{i_{N-1}} \mid\left(i_{0}, \ldots, i_{N-1}\right) \in S\left(k^{N-j},(k+1)^{j}\right)\right\} .
$$


Remark. The fusion product $V(1)^{* N}$ is isomorphic to the truncated Weyl module $W_{\text {loc }}(N, N)$ and also to the local Weyl module $W_{\text {loc }}(N)$. The inductively obtained basis $B\left(1^{N}\right)$ coincides with the basis of the Weyl module $W_{\text {loc }}(N)$ constructed in [Chari and Pressley 2001]. However, we would like to emphasize that the PBW type basis of the truncated Weyl module $\mathrm{W}(m, N)$ described in Theorem 4.3 is different from the basis described in [Chari and Venkatesh 2015, Section 6]. For example, we have $f_{1}^{3} \in B\left(1^{4}\right)$ but $f_{1}^{3}$ is not contained in their basis.

\section{References}

[Ardonne and Kedem 2007] E. Ardonne and R. Kedem, "Fusion products of Kirillov-Reshetikhin modules and fermionic multiplicity formulas", J. Algebra 308:1 (2007), 270-294. MR 2008a:17028 Zbl 1122.17014

[Chari and Loktev 2006] V. Chari and S. Loktev, "Weyl, Demazure and fusion modules for the current algebra of $\mathfrak{s l}_{r+1}$ ”, Adv. Math. 207:2 (2006), 928-960. MR 2008a:17029 Zbl 1161.17318

[Chari and Pressley 2001] V. Chari and A. Pressley, "Weyl modules for classical and quantum affine algebras", Represent. Theory 5 (2001), 191-223. MR 2002g:17027 Zbl 0989.17019

[Chari and Venkatesh 2015] V. Chari and R. Venkatesh, "Demazure modules, fusion products and Q-systems”, Comm. Math. Phys. 333:2 (2015), 799-830. MR 3296163 Zbl 06394855

[Chari et al. 2014a] V. Chari, G. Fourier, and D. Sagaki, "Posets, tensor products and Schur positivity", Algebra Number Theory 8:4 (2014), 933-961. MR 3248990 Zbl 06331627

[Chari et al. 2014b] V. Chari, B. Ion, and D. Kus, "Weyl modules for the hyperspecial current algebra", Int. Math. Res. Not. (online publication August 2014).

[Chari et al. 2014c] V. Chari, P. Shereen, R. Venkatesh, and J. Wand, "A Steinberg type decomposition theorem for higher level Demazure modules", preprint, 2014. arXiv 1408.4090

[Feigin 2008] E. Feigin, "The PBW filtration, Demazure modules and toroidal current algebras", SIGMA Symmetry Integrability Geom. Methods Appl. 4 (2008), Paper 070. MR 2010b:17029 Zbl 1215.17015

[Feigin and Feigin 2002] B. Feigin and E. Feigin, " $Q$-characters of the tensor products in $\mathfrak{s l}_{2}$-case", Mosc. Math. J. 2:3 (2002), 567-588. MR 2004e:17002 Zbl 1027.05007

[Feigin and Loktev 1999] B. Feigin and S. Loktev, "On generalized Kostka polynomials and the quantum Verlinde rule”, pp. 61-79 in Differential topology, infinite-dimensional Lie algebras, and applications, edited by A. Astashkevich and S. Tabachnikov, Amer. Math. Soc. Transl. Ser. 2 194, Amer. Math. Soc., Providence, RI, 1999. MR 2002b:17007 Zbl 0974.17008

[Feigin et al. 2004] B. Feigin, M. Jimbo, R. Kedem, S. Loktev, and T. Miwa, "Spaces of coinvariants and fusion product, II: $\widehat{\mathfrak{s l}}_{2}$ character formulas in terms of Kostka polynomials", J. Algebra 279:1 (2004), 147-179. MR 2005m:17033 Zbl 1160.17312

[Fourier 2015] G. Fourier, "New homogeneous ideals for current algebras: filtrations, fusion products and Pieri rules", Mosc. Math. J. 15:1 (2015), 49-72.

[Fourier and Kus 2013] G. Fourier and D. Kus, "Demazure modules and Weyl modules: the twisted current case”, Trans. Amer. Math. Soc. 365:11 (2013), 6037-6064. MR 3091275 Zbl 1287.17016

[Fourier and Littelmann 2006] G. Fourier and P. Littelmann, "Tensor product structure of affine Demazure modules and limit constructions", Nagoya Math. J. 182 (2006), 171-198. MR 2007e:17021 Zbl 1143.22010 
[Fourier and Littelmann 2007] G. Fourier and P. Littelmann, "Weyl modules, Demazure modules, KR-modules, crystals, fusion products and limit constructions", Adv. Math. 211:2 (2007), 566-593. MR 2008k:17005 Zbl 1114.22010

[Fourier et al. 2012] G. Fourier, T. Khandai, D. Kus, and A. Savage, "Local Weyl modules for equivariant map algebras with free abelian group actions", J. Algebra 350 (2012), 386-404. MR 2859894 Zbl 1268.17022

[Garland 1978] H. Garland, "The arithmetic theory of loop algebras", J. Algebra 53:2 (1978), 480551. MR 80a:17012 Zbl 0383.17012

[Kac 1990] V. G. Kac, Infinite-dimensional Lie algebras, 3rd ed., Cambridge University Press, Cambridge, 1990. MR 92k:17038 Zbl 0716.17022

[Kedem 2011] R. Kedem, "A pentagon of identities, graded tensor products, and the KirillovReshetikhin conjecture", pp. 173-193 in New trends in quantum integrable systems, edited by B. Feigin et al., World Sci. Publ., Hackensack, NJ, 2011. MR 2012g:17011 Zbl 1221.82037

[Kus and Venkatesh 2014] D. Kus and R. Venkatesh, "Twisted Demazure modules, fusion product decomposition and twisted $Q$-systems”, preprint, 2014. arXiv 1409.1201

[Mathieu 1988] O. Mathieu, "Construction du groupe de Kac-Moody et applications", C. R. Acad. Sci. Paris Sér. I Math. 306:5 (1988), 227-230. MR 89e:17013 Zbl 0666.17006

[Naoi 2012] K. Naoi, "Weyl modules, Demazure modules and finite crystals for non-simply laced type”, Adv. Math. 229:2 (2012), 875-934. MR 2855081 Zbl 1305.17009

[Ravinder 2014] B. Ravinder, "Demazure modules, Chari-Venkatesh modules and fusion products”, SIGMA Symmetry Integrability Geom. Methods Appl. 10 (2014), Paper 110. MR 3298990 Zbl 06421406

Received November 26, 2014. Revised March 18, 2015.

\section{DENIZ KUS}

Mathematical Institute of The University of Cologne

D-50931 COLOGNE

GERMANY

dkus@math.uni-koeln.de

Peter LitTelmanN

Mathematical Institute of The University of Cologne

D-50931 COLOGNE

GERMANY

peter.littelmann@math.uni-koeln.de 


\title{
PACIFIC JOURNAL OF MATHEMATICS
}

\author{
msp.org/pjm
}

Founded in 1951 by E. F. Beckenbach (1906-1982) and F. Wolf (1904-1989)

\section{EDITORS}

Don Blasius (Managing Editor)

Department of Mathematics

University of California

Los Angeles, CA 90095-1555

blasius@math.ucla.edu

\author{
Paul Balmer \\ Department of Mathematics \\ University of California \\ Los Angeles, CA 90095-1555 \\ balmer@math.ucla.edu \\ Robert Finn \\ Department of Mathematics \\ Stanford University \\ Stanford, CA 94305-2125 \\ finn@math.stanford.edu \\ Sorin Popa \\ Department of Mathematics \\ University of California \\ Los Angeles, CA 90095-1555 \\ popa@math.ucla.edu
}

\author{
Vyjayanthi Chari \\ Department of Mathematics \\ University of California \\ Riverside, CA 92521-0135 \\ chari@math.ucr.edu \\ Kefeng Liu \\ Department of Mathematics \\ University of California \\ Los Angeles, CA 90095-1555 \\ liu@math.ucla.edu \\ Jie Qing \\ Department of Mathematics \\ University of California \\ Santa Cruz, CA 95064 \\ qing@ cats.ucsc.edu
}

\section{PRODUCTION}

Silvio Levy, Scientific Editor, production@msp.org

\section{SUPPORTING INSTITUTIONS}

ACADEMIA SINICA, TAIPEI

CALIFORNIA INST. OF TECHNOLOGY

INST. DE MATEMÁTICA PURA E APLICADA

KEIO UNIVERSITY

MATH. SCIENCES RESEARCH INSTITUTE

NEW MEXICO STATE UNIV.

OREGON STATE UNIV.

\author{
STANFORD UNIVERSITY \\ UNIV. OF BRITISH COLUMBIA \\ UNIV. OF CALIFORNIA, BERKELEY \\ UNIV. OF CALIFORNIA, DAVIS \\ UNIV. OF CALIFORNIA, LOS ANGELES \\ UNIV. OF CALIFORNIA, RIVERSIDE \\ UNIV. OF CALIFORNIA, SAN DIEGO \\ UNIV. OF CALIF., SANTA BARBARA
}

\author{
Daryl Cooper \\ Department of Mathematics \\ University of California \\ Santa Barbara, CA 93106-3080 \\ cooper@math.ucsb.edu \\ Jiang-Hua Lu \\ Department of Mathematics \\ The University of Hong Kong \\ Pokfulam Rd., Hong Kong \\ jhlu@maths.hku.hk \\ Paul Yang \\ Department of Mathematics \\ Princeton University \\ Princeton NJ 08544-1000 \\ yang@math.princeton.edu
}

These supporting institutions contribute to the cost of publication of this Journal, but they are not owners or publishers and have no responsibility for its contents or policies.

See inside back cover or msp.org/pjm for submission instructions.

The subscription price for 2015 is US \$420/year for the electronic version, and \$570/year for print and electronic.

Subscriptions, requests for back issues and changes of subscribers address should be sent to Pacific Journal of Mathematics, P.O. Box 4163, Berkeley, CA 94704-0163, U.S.A. The Pacific Journal of Mathematics is indexed by Mathematical Reviews, Zentralblatt MATH, PASCAL CNRS Index, Referativnyi Zhurnal, Current Mathematical Publications and Web of Knowledge (Science Citation Index).

The Pacific Journal of Mathematics (ISSN 0030-8730) at the University of California, c/o Department of Mathematics, 798 Evans Hall \#3840, Berkeley, CA 94720-3840, is published twelve times a year. Periodical rate postage paid at Berkeley, CA 94704, and additional mailing offices. POSTMASTER: send address changes to Pacific Journal of Mathematics, P.O. Box 4163, Berkeley, CA 94704-0163.

PJM peer review and production are managed by EditFLOW ${ }^{\circledR}$ from Mathematical Sciences Publishers.

\section{PUBLISHED BY}

\section{mathematical sciences publishers \\ nonprofit scientific publishing}

http://msp.org/

(C) 2015 Mathematical Sciences Publishers 


\section{PACIFIC JOURNAL OF MATHEMATICS}

Volume $278 \quad$ No. $2 \quad$ December 2015

Differential Harnack and logarithmic Sobolev inequalities along

Ricci-harmonic map flow

ABIMBOLA ABOLARINWA

On $J$-holomorphic curves in almost complex manifolds with

291

asymptotically cylindrical ends

ERKAO BAO

Integration of coupling Dirac structures

OLIVIER BRAHIC and RUI LOJA FERNANDES

Asymptotic behavior of Palais-Smale sequences associated with fractional Yamabe-type equations

Yi FANG and MARÍA DEL MAR GONZÁLEZ

$K$-theory and homotopies of 2-cocycles on higher-rank graphs

ELIZABETH GILLASPY

Fusion products and toroidal algebras

DENIZ KUS and PETER LiTTELMANN

Differential Harnack estimates for positive solutions to heat equation under Finsler-Ricci flow

\section{SAJJAD LAKZIAN}

On the one-endedness of graphs of groups

NichOLAS TOUIKAN

On the structure of vertex cuts separating the ends of a graph

GARETH R. WILKES 